\title{
Suspensión de la anticoagulación oral en pacientes con fibrilación auricular ante un procedimiento invasivo. ¿Deben recibir un puenteo con anticoagulación parenteral durante su realización?
}

Suspension of oral anticoagulation in patients with atrial fibrillation before an invasive procedure. Should they receive parenteral anticoagulation bridging during its implementation?

María Paula Cárdenas* y Esteban Gabriel Jauregui **

\section{Resumen}

En la actualidad, muchos pacientes con fibrilación auricular son anticoagulados por largos períodos. Durante este tiempo pueden ser sometidos a procedimientos invasivos. A partir de una viñeta clínica, un médico se plantea el impacto de utilizar o no heparina de bajo peso molecular como puente farmacológico al momento de suspender la anticoagulación oral. Luego de realizar una búsqueda ad hoc, un ensayo clínico aleatorizado de no inferioridad demuestra que en pacientes con fibrilación auricular, con puntajes de riesgo tromboembólico $\left(\mathrm{CHADS}_{2}\right)$ intermedios a bajos que requieren una interrupción temporal del tratamiento con warfarina para un procedimiento invasivo electivo, la estrategia de no reemplazar la anticoagulación oral con heparina de bajo peso molecular no resultó inferior (o menos efectiva) para la prevención de tromboembolismo arterial, y disminuyó además el riesgo de sangrado mayor en comparación al uso de un puente con esta medicación.

\begin{abstract}
Many patients with atrial fibrillation are anticoagulated for long periods. During this time they may be subjected to invasive procedures. From a clinical vignette, a physician discusses the impact of using (or not) low molecular weight heparin as a pharmacological bridge at the time of suspending oral anticoagulation. After conducting a bibliographic search, a no inferiority randomized clinical trial showed that in patients with atrial fibrillation with intermediate to low thromboembolic risk $\left(\mathrm{CHADS}_{2}\right.$ ) requiring a temporary interruption of warfarin therapy for an elective invasive procedure, the strategy of withholding low molecular weight heparin bridging was not inferior (or less effective) for the prevention of arterial thromboembolism than its use, also decreasing the risk of major bleeding.
\end{abstract}

Cardenas MP, Jauregui EG. Suspensión de la anticoagulación oral en pacientes con fibrilación auricular ante un procedimiento invasivo. ¿Deben recibir un puenteo con anticoagulación parenteral durante su realización? Evid Act Pract Ambul 2017;20(2):59-60.

\begin{abstract}
Escenario clínico
Una paciente de 50 años acude al consultorio de su médico de cabecera. Recibe la indicación de realizar una videocolonoscopía (VCC) de rastreo. Tiene una fibrilación auricular (FA) crónica, por la que está anticoagulada con acenocumarol. El profesional le indica que suspenda la anticoagulación desde los cinco días anteriores al procedimiento, iniciando heparina de bajo peso molecular dos días antes de realizarlo.
\end{abstract}

Anticoagulación en pacientes sometidos a un procedimiento invasivo

En la actualidad, muchos pacientes con FA son anticoagulados por largos períodos ${ }^{1}$. Durante el tiempo en que están anticoagulados pueden ser sometidos a procedimientos invasivos. Estos pueden ser desde simples o de bajo riesgo (p. ej. videocolonoscopía), hasta complejos y de alto riesgo (p. ej. cirugía mayor).

Generalmente, los pacientes con indicación de algún procedimiento programado, son sometidos a un puente en la anticoagulación ${ }^{2}$. Es decir, suspenden la anticoagulación oral (que requiere de varios días para poder revertir su efecto) y, luego, empiezan su profilaxis con anticoagulantes parenterales (ge-neralmente heparina de bajo peso molecular [HBPM], que tiene un tiempo de reversión más corto). Esta situación afecta, aproximadamente, a uno de cada seis pacientes anticoagulados con FA. La anticoagulación parenteral tiene el objetivo de minimizar posibles eventos tromboembólicos, como el accidente cerebrovascular (ACV).

Múltiples estudios observacionales han evaluado el timing y la dosificación necesaria para este puenteo perioperatorio. Sin embargo, la pregunta de si la anticoagulación parenteral como puente es necesaria, continua vigente. Justamente debido a este hecho, las guías de práctica clínica no han realizado recomendaciones fuertes al respecto.

\section{Pregunta que generó el caso}

En pacientes con fibrilación auricular que están recibiendo anticoagulantes orales (población), ¿cambiar temporariamente la anticoagulación oral hacia parenteral antes de un procedimiento invasivo (intervención) versus únicamente suspender la anticoagulación oral (comparación), reduce la incidencia de eventos tromboembólicos (resultado)?

\section{Estrategia de búsqueda}

Se realizó una búsqueda bibliográfica en las bases de datos PubMed, The Cochrane Library y TripDataBase utilizando como palabras clave oral anticoagulant, bridging, heparin, invasive procedure. La búsqueda arrojó sólo un trabajo prospectivo que había comparado la incidencia de eventos tromboembólicos y hemorrágicos en pacientes sometidos a un procedimiento invasivo.

\section{Resumen de la evidencia}

Douketis JD, y col. Perioperative Bridging Anticoagulation in Patients with Atrial Fibrillation. N Engl J Med 2015;373:823-33³.

Objetivo: Comparar la eficacia (protección de eventos tromboembólicos), y seguridad (aparición de sangrados) de utilizar o no un puente con HBPM durante la suspensión de los dicumarínicos en pacientes anticoagulados que serán sometidos a procedimientos invasivos.

Diseño y lugar y pacientes: Ensayo clínico aleatorizado, doble ciego, controlado con placebo, de no inferioridad; realizado en Canadá y Estados Unidos.

Incluyó adultos mayores de 18 años con aleteo o FA, permanente o paroxística, anticoagulados con warfarina por tres meses o más, en rango terapéutico (RIN entre 2 y 3 ) que se encontraban en plan de realizarse un procedimiento invasivo electivo. Debían tener al menos un factor de riesgo en la escala CHADS $_{2}$ : insuficiencia cardiaca congestiva o disfunción ventricular izquierda, hipertensión arterial, edad mayor o igual a 75 años, diabetes, $\mathrm{ACV}$ isquémico previo, embolia sistémica o accidente isquémico transitorio (AIT). No fueron elegibles los pacientes con: reemplazo valvular mecánico, embolia sistémica en los 12 meses pre-

*Sección Hematología, Servicio de Clínica Médica del Hospital Italiano de Buenos Aires. maria.cardenas@ @ospitalitaliano.org.ar

** Servicio de Anestesiología del Hospital Italiano de Buenos Aires. esteban.jauregui@ hiba.org.ar 
vios, sangrado mayor en las doce semanas previas, clearance de creatinina menor de $30 \mathrm{ml}$, recuento de plaquetas menor de $100.000 / \mathrm{mm}^{3}$, cirugía programada cardíaca, intracraneal o intraespinal.

Intervención: La warfarina se discontinuaba cinco días antes del procedimiento, para reiniciarse el día del mismo. Los pacientes fueron aleatorizados (relación 1:1) a recibir puente con dalteparina sódica (100 U/kg cada $12 \mathrm{hs}$ ) o placebo desde los tres días previos al procedimiento, y hasta cinco a diez días posteriores al mismo, una vez obtenido un RIN en rango terapéutico. Se realizó un seguimiento telefónico a las cinco semanas de finalizado el procedimiento.

Medición de resultados principales: Tromboembolismo arterial
(ACV isquémico o hemorrágico, AIT, embolia sistémica) y sangrado mayor a los 30 días. El análisis que se utilizó fue de no inferioridad. Fueron resultados secundarios infarto agudo de miocardio, trombosis venosa profunda, embolia pulmonar, muerte y sangrado menor.

Resultados Principales: Fueron reclutados 1.884 pacientes, 950 de los cuales fueron asignados a la rama placebo y 934 a la de tratamiento con dalteparina. La edad media fue de 72 años (73\% varones). El $\mathrm{CHADS}_{2}$ promedio fue de 2,3 , y el $38 \%$ de los pacientes tenían un CHADS 2 mayor de tres. Los procedimientos más frecuentemente indicados fueron los de origen gastrointestinal. A su vez, un $89 \%$ de los pacientes fueron sometidos a un procedimiento menor, sin embargo, solo un $69 \%$ fue tratado como si hubieran tenido un riesgo bajo de sangrado.

Tabla 1. Incidencia de tromboembolismo arterial y sangrado mayor según grupo a los 30 días del procedimiento invasivo.

\begin{tabular}{|c|c|c|c|c|}
\hline Resultados & $\begin{array}{l}\text { Grupo placebo } \\
(\mathrm{N}=918)\end{array}$ & $\begin{array}{c}\text { Grupo puente } \\
(\mathrm{N}=895)\end{array}$ & $\begin{array}{l}\text { Medida de eficacia o } \\
\text { seguridad }(\text { (C95\%) }\end{array}$ & $\mathbf{P}$ \\
\hline Tromboembolismo arterial, $\mathrm{n}(\%)$ & $4(0,4)$ & $3(0,3)$ & $0,1 \%{ }^{*}(-0,6$ a 0,8$)$ & $0,01^{\star *} ; 0,73^{t+}$ \\
\hline Sangrado mayor, n (\%) & $12(1,3)$ & $29(3,2)$ & $0,41 \dagger(0,20$ a 0,78$)$ & $0,005^{+\dagger}$ \\
\hline
\end{tabular}

RRA: reducción de riesgo absoluto. IC95\%: intervalo de confianza del $95 \%$. ${ }^{*}$ Reducción de riesgo absoluto. ${ }^{\dagger}$ Riesgo relativo. ${ }^{* *}$ valor de $\mathrm{P}$ no-inferioridad. "t valor de $\mathrm{P}$ superioridad.

Los resultados principales se resumen en la tabla 1.

Ningún sangrado mayor fue fatal. Con respecto al sangrado menor, el grupo placebo tuvo una incidencia de $12 \%$ versus $20,9 \%$ del grupo dalteparina $(P<0,001)$. No hubo diferencias significativas con respecto al infarto agudo de miocardio, trombosis venosa profunda, embolia pulmonar o muerte.
Conclusiones: En pacientes con FA que requieren una interrupción temporal del tratamiento con warfarina para un procedimiento invasivo electivo, la estrategia de no reemplazar la warfarina con HBPM no resultó inferior (o menos efectiva) para la prevención de tromboembolismo arterial que el uso de un puente con HBPM, y disminuyó además el riesgo de sangrado mayor.

\section{Comentario}

Los hallazgos de estos estudios son consistentes con otros estudios no aleatorizados. En un metaanálisis con 12.278 pacientes con FA o reemplazo valvular mecánico que recibieron o no HBPM como puente perioperatorio, no se observaron diferencias en la tasa de tromboembolismo arterial (odds ratio 0,80 ; IC95\% 0,42 a 1,54) pero sí mayor sangrado $\left(3,60 ; 1,52\right.$ a 8,50) ${ }^{4}$. Sin embargo, este estudio tuvo algunas limitaciones que discutiremos a continuación. En primer lugar, hay ciertos grupos poblacionales, como los pacientes con un puntaje $\mathrm{CHADS}_{2}$ de 5 ó 6 , o los sometidos a procedimientos invasivos muy relacionados con eventos tromboembólicos (endarterectomía carotídea, cirugía oncológica mayor, cirugía cardíaca o neurológica), que no fueron adecuadamente representados. Cabe señalar nuevamente, que los procedimientos más representados fueron, también, los más comúnmente realizados en la población general (ej. colonoscopia). La media del CHADS fue de 2,3 puntos, similar a la mayoría de los estudios recientes ${ }^{5,6}$. En segundo lugar, la tasa global de eventos tromboembólicos arteriales fue menor a la esperada durante el cálculo del tamaño muestral (0,4 vs. $1 \%)$, lo que podría haber afectado los cálculos de poder para detectar los eventuales beneficios de realizar el puenteo. Por otro lado, se puede argumentar en contra de este estudio el hecho de que la medicación antiplaquetaria era manejada según los criterios

Fuente de financiamiento: National Heart, Lung, and Blood Institute.

específicos de cada centro investigador y no con una sistemática previamente estipulada. Esto podría sesgar a las mediciones efectuadas, aunque no parece haber habido diferencias significativas a este respecto entre las dos poblaciones del ensayo. Finalmente, se podría decir que en la argentina el uso de warfarina no está muy difundido y que el acenocumarol es la droga de elección en estos casos. Sin embargo, en términos de eficacia, cinética y perfil de efectos adversos son drogas muy similares, haciendo posible la extrapolación de datos de estudios hechos con una droga a la otra. Mención aparte merecen los "nuevos" anticoagulantes, especialmente los inhibidores directos de la trombina, para los cuales son necesarios más estudios clínicos, pero debido a su corta vida media es probable que no sea necesario el uso de HBPM.

\section{Conclusiones de los comentadores}

En la práctica cotidiana, cuando es necesario revertir el tratamiento anticoagulante en pacientes con FA no valvular para procedimientos invasivos, es recomendable suspender los dicumarínicos sin terapia puente, en aquellos pacientes con $\mathrm{CHADS}_{2}$ bajo (entre 2 y 3 ). En los pacientes con $\mathrm{CHADS}_{2} \geq 4$, se sugiere realizar terapia puente adecuando las dosis de HBPM al riesgo de sangrado y al tipo de procedimiento.

\section{Referencias}

Recibido el 02/05/16 y aceptado el 01/09/17.

1. Fuster $\mathrm{V}$ y col. $2011 \mathrm{ACCF} / \mathrm{AHA} / \mathrm{HRS}$ focused updates incorporated into the ACC/AHA/ESC 2006 Guidelines for the management of patients with atrial fibrilation: a report of the American College of Cardiology Foundation/American Heart Association Task Force on Practice Guidelines developed in partnership with the European Society of Cardiology and in collaboration with the European Heart Rythm Association and Heart Rythm Society. J Am Coll Cardiol. 2011 Mar 15;57(11):e101-98.

2. Douketis J y col. Perioperative management of antithrombotic therapy: Antithrombotic Therapy and Prevention of Thrombosis, 9th ed: American College of Chest Physicians Evidence-Based Clinical Practice Guidelines. Chest 2012 Feb;141(2 Suppl):e326S-e350S.

3. Douketis JD, y col. Perioperative Bridging Anticoagulation in Patients with Atrial Fibrillation. N Engl J Med 2015;373:823-33. PMID: 26095867.

4. Siegal D, y col. Periprocedural heparin bridging in patients receiving vita- min K antagonists: systematic review and meta-analysis of bleeding and thromboembolic rates. Circulation 2012;126:1630-9.

5. Granger CB, y col. Apixaban versus warfarin in pa- tients with atrial fibrillation. N Engl J Med 2011;365:981-92.

6. Graham DJ, y col. Cardiovascular, bleeding, and mortality risks in elderly Medicare patients treated with dabigatran or warfarin for nonvalvular atrial fibrillation. Circulation $2015 ; 131: 157-64$. 